\title{
Novel metaphor translation is modulated by translation direction
}

\author{
Katarzyna Jankowiak* (iD and Olha Lehka-Paul \\ Faculty of English, Adam Mickiewicz University, Poznan, Poland \\ ${ }^{*}$ Corresponding author. Email: katarzyna.jankowiak@amu.edu.pl
}

(Received 23 September 2020; revised 20 August 2021; accepted 26 August 2021; first published online 09 November 2021)

\begin{abstract}
Previous translation process research has pointed to an increased cognitive load when translating metaphoric compared to literal language. Yet, studies conducted thus far have not examined the role of translation direction (i.e., L1-L2 vs. L2-L1) in novel metaphor translation and have not tested whether and how this process might be modulated by the linguistic form of a novel meaning. In the present study, Polish (L1) - English (L2) translation students translated novel nominal metaphors $(A$ is $B$ ), novel similes ( $A$ is like $B$ ), and literal sentences, in either L1-L2 or L2-L1 translation directions, while their translation behavior was recorded using a keystroke logging method. The results revealed longer translation durations for both metaphors and similes relative to literal utterances. Furthermore, we found slower translation times for novel nominal metaphors compared to novel similes and literal sentences, yet only in the L2-L1 translation direction. Such results might indicate that novel meaning translation is more cognitively taxing in the case of novel nominal metaphors, which require a more robust activation of comparison mechanisms, relative to novel similes. Importantly, this effect might be stronger when translating in the direction in which access to semantic representations is potentially more automatic (i.e., L2-L1 translation).
\end{abstract}

Keywords: translation process; novel metaphor; novel simile; translation directionality; keystroke logging

Previous research has repeatedly indicated that metaphor comprehension is more cognitively taxing in the non-native (L2) relative to the native (L1) tongue, as evidenced by longer reaction times (RTs), lower accuracy rates, and more extended meaning integration mechanisms observed in electrophysiological (EEG) patterns (e.g., Heredia \& Cieślicka, 2016; Jankowiak, 2019; Vaid et al., 2015; Wang \& Jankowiak, 2021). At the same time, translation process research has suggested that metaphor translation is even more challenging than metaphor comprehension itself, as it requires the additional process of re-mapping (Massey, 2016) that involves not only the processes of cross-domain mappings necessary for metaphor comprehension but also the translation of this mapping into a target language (Jakobsen et al.,

(c) The Author(s), 2021. Published by Cambridge University Press. This is an Open Access article, distributed under the terms of the Creative Commons Attribution licence (http://creativecommons.org/licenses/by/4.0/), which permits unrestricted re-use, distribution and reproduction, provided the original article is properly cited. 
2007; Massey, 2016; Massey \& Ehrensberger-Dow, 2017; Schäffner, 2004). It, however, remains under-investigated whether the cognitive effort invested in novel figurative meaning translation might be modulated by a linguistic structure in which a figure of speech is presented. Also, only a little attention has been devoted to examining how translation direction might influence the time course of nonliteral meaning translation. The present study is aimed to test whether and how the translation process is modulated by the type of a novel nonliteral utterance (i.e., novel nominal metaphor vs. novel simile), and whether it might be additionally dependent on translation direction (i.e., L1-L2 vs. L2-L1).

Metaphors, defined as utterances whose comprehension requires cross-domain mappings between two presumably distinct concepts (Gibbs \& Colston, 2012), vary along their conventionality continuum. While the links between metaphor source and target domains of conventional (familiar) metaphors have become lexicalized as a result of its frequency of use, the semantic category denoting a nonliteral meaning of novel (unfamiliar and highly creative) metaphors has not been yet established (Bowdle \& Gentner, 2005). The cognitive mechanisms engaged in novel and conventional metaphor processing have been explicated within, for instance, the Career of Metaphor Model (Bowdle \& Gentner, 2005), according to which while conventional metaphors are preferentially processed as categorizations, novel metaphors are comprehended by means of comparison mechanisms, whereby the two metaphor domains have to be structurally aligned. Importantly, such comparison processes engaged in novel metaphor comprehension are hypothesized to be facilitated when a novel meaning has a form of a simile ( $A$ is like $B)$, which automatically initiates comparison mechanisms. This assumption has been corroborated in previous behavioral as well as brain imaging studies (e.g., Bowdle \& Gentner, 2005; Jankowiak, 2019; Jankowiak et al., 2021; Lai \& Curran, 2013; Shibata et al., 2012). For example, in a monolingual context, Shibata et al. (2012) employed the functional magnetic resonance imaging (fMRI) and showed that novel meanings are perceived by the brain as more salient when presented as similes relative to nominal metaphors ( $A$ is $B$ ). More recently, Jankowiak et al. (2021) examined bilingual novel meaning processing in an event-related potential (ERP) study and found a facilitation effect for novel similes, relative to novel nominal metaphors, within the time window of the late positive complex (LPC) in both L1 and L2, which suggests a continuous difficulty in novel nominal metaphor integration, irrespective of language nativeness.

Thus far, however, novel metaphor translation has received little scholarly attention. Importantly, cognitive mechanisms engaged in meaning comprehension tasks differ to a considerable extent from processes involved in translation. Namely, while the aforementioned studies mostly tapped into such mechanisms as visual processing, lexico-semantic information retrieval, and meaning integration, all of which are assumed as rather automatic, translation tasks additionally require complex conscious mechanisms, such as information processing, decision-making and problem solving, meaning construction, attentional control, and self-monitoring. Hence, studies into novel metaphor translation might offer an innovative insight into novel meaning processing in a complex set of conscious cognitive mechanisms involved in translation.

In translation studies, metaphor translation has often been addressed in productoriented research, whereby the issues of translatability and creative shifts employed 
by translators have been analyzed (Bayer-Hohenwarter, 2009; Göpferich, 2008; Schäffner, 2004; Toury, 1995). In studies on translation process, much scholarly attention has been devoted to employing empirical research methods, such as eye-tracking and keylogging, to investigate the problem of metaphors translation. For instance, using the eye-tracking method, which measures eye positions and eye movements in response to a given word or area of interest (Conklin \& Pellicer-Sánchez, 2016), Sjørup (2008) found longer fixation times to metaphors relative to literal items when translating, thus pointing to an increased cognitive load engaged in metaphor translation. Similar to eye-tracking experiments, studies using the keylogging technique have also indicated that metaphor translation is a more difficult task for translators compared to literal language translation. The keystroke logging technique allows for tracking and recording the process of translation as it occurs on a computer screen in real time. In addition to the function of post-task replay of the text production process, keyloggers also trace all revisions (deletions, additions, and substitutions) and measure task time as well as the duration and number of pauses. In one of the keylogging studies, Jakobsen et al. (2007) observed slower translation processes in response to figurative items provided within a longer passage to be translated. Recently, Lehka-Paul (2020) used the keylogging method (Translog-II; Carl, 2012) and found that translators introduced more revisions and spent more time on revising the first draft of the target text when translating expressive texts, that is, those that "communicate an artistically organized content" (Reiss, 2000 , p. 161) than informative texts, that is, those that "communicate content" (ibid.). This finding suggests that at the stage of target text production, the texts that contain figurative expressions are more challenging for translators in comparison to those that use plain language. On a similar note, combining the methods of eye-tracking and keylogging, Sjørup (2013) found that the translation process is more effortful and time-consuming in metaphor-laden as opposed to literal content. Furthermore, Sjørup (2013) observed faster metaphor processing in reading for comprehension as compared to reading for translation task, thus indicating that metaphor processing is modulated by task type. Altogether, available research into metaphor translation has shown that translation time may be one of the potential indicators of increased cognitive load involved in the translation of metaphorical expressions relative to literal utterances. Yet, to the best of our knowledge, previous studies have not addressed novel metaphor translation and have not been aimed to show whether metaphor translation might be modulated by the translation direction (i.e., L1-L2 vs. L2-L1 translation).

General differences in translation mechanisms as modulated by translation direction were explicated in the Revised Hierarchical Model (RHM; Kroll \& Stewart, 1994), which postulates that the bilingual lexicon comprises lexical and conceptual representations, and consequently any lexical item has separate representations for word form and word meaning. Since L1 lexicon is assumed to be more strongly interconnected (Potter et al., 1984), access to a lexical form is more automatic in L1 compared to L2. Furthermore, lexical-level representations are assumed to have stronger connections with conceptual-level representations in the native relative to the non-native language, and consequently, links between the two languages are weaker and less automatic in the L1-L2 than in the L2-L1 direction. Based on all these assumptions, the RHM postulates a disadvantage of the forward (L1-L2) 
translation direction, whereby access to semantic representations is less privileged. Importantly, the model assumes that such a difference between the two translation directions is observed mostly in non-proficient bilinguals, as the disparity between L1 and L2 lexicons attenuates in highly proficient bilingual speakers.

Similarly, L2-L1 translation direction has been widely regarded as more natural and less cognitively taxing in translation studies (Newmark, 1988). Kelly (2005) argued that there is a comprehension disadvantage when the source text is in the participants' L2, and production advantage is mainly related to language proficiency when producing the target text in the participants' L1, which is in line with the Revised Hierarchical Model (Kroll \& Stewart, 1994). Previous research has also shown that L2-L1 translation takes less time than L1-L2 translation (Buchweitz \& Alves, 2006; Ferreira, 2012; Pavlović, 2007; Pavlović \& Jensen, 2009), which suggests that translation time may be an indicator of cognitive effort involved in L1-L2 vs. L2-L1 translation. However, a more granular analysis performed by means of data triangulation employing the methods of eye-tracking, keylogging, and retrospection indicates that the results obtained by other measures, such as gaze time, total fixation durations, or revision rates (Fonseca, 2015; Pavlović \& Jensen, 2009; Whyatt 2019), challenge the claim that L1-L2 translation is indeed more cognitively demanding than L2-L1 translation. Interestingly, da Silva et al. (2017) and Whyatt (2019) reported that in the groups of professional translators, total task time was not found to be indicative of an increased cognitive effort regardless of translation direction, thus suggesting that both directions can be equally demanding for professional translators. Importantly, as the aforementioned studies were conducted using texts that varied in length, genres, and readability levels, the provided context might have influenced the obtained results. The current study aims to eliminate a potential effect of this variable by means of using single sentences as the stimuli. Also, it remains an open question whether translation direction modulates novel simile and novel nominal metaphor translation, as reflected in keystroke patterns.

The present study aims to provide insights into the keystroke correlates of novel metaphor translation and to investigate the role of translation direction (i.e., L1-L2 vs. L2-L1 translation directions) in this process. Additionally, the study aims to exploratively examine the potential role of a linguistic structure of a novel meaning (i.e., novel nominal metaphors vs. novel similes) in the process of translation. To this end, employing a keylogging method, proficient Polish (L1) - English (L2) translation students were asked to translate novel nominal metaphors, novel similes, and literal sentences (a within-subject variable) in the two translation directions (a between-subject variable). First, due to the fact that both novel similes and novel nominal metaphors require more extended semantic processes that include the detection of semantic deviation (Hagoort et al., 2004; Ni et al., 2000; Shibata et al., 2012) as well as mapping between dissimilar concepts, we hypothesize longer translation times for both novel similes and novel nominal metaphors than literal sentences, which on the other hand require mapping between similar concepts (Lai \& Curran, 2013). Such findings would be in line with previous studies on nonliteral meaning translation using the keylogging technique (e.g., Jakobsen et al., 2007; Lehka-Paul, 2020) and would extend research on simile and metaphor processing from studying mostly automatic comprehension processes (Bowdle \& Gentner, 2005; Jankowiak, 2019; Jankowiak et al., 2021; Lai \& Curran, 2013; Shibata et al., 
2012) to the investigation of the conscious process of translation. Second, since nominal metaphors ( $A$ is $B$ ), unlike similes $(A$ is like $B)$, need to be converted into a comparison form to facilitate comparison mechanisms (Bowdle \& Gentner, 2005), this might be reflected in faster translation of similes ( $A$ is like $B$ ) compared to nominal metaphors ( $A$ is $B$ ), even though the two expressions share the same source and target domain and are high in novelty, thus requiring mapping between highly dissimilar concepts. However, it remains an open question whether the aforementioned effects would be observed in both translation directions. Namely, in line with the Revised Hierarchical Model (Kroll \& Stewart, 1994), there is a disadvantaged access to semantic representations in the L1-L2 compared to L2-L1 translation direction. Consequently, there may be a facilitation in simile translation only in the L2-L1 direction. In the L1-L2 translation direction, on the other hand, no differences in time needed to translate similes vs. nominal metaphors would be suggestive of a generally more cognitively taxing mechanisms engaged in lexico-semantic access in L1-L2, irrespective of utterance type. Alternatively, if the effect of translation direction is modulated by L2 proficiency level, similes might be easier to translate than nominal metaphors, irrespective of translation direction.

\section{Method}

\section{Participants}

The original sample included 23 participants, but one of them was removed from further analyses due to missing values in the exported data, and another one was identified as an outlier (Stevens, 2009). This resulted in a final sample of 21 participants ( 14 women, $M_{\text {age }}=21.00, S D=1.55$ ), who were all 2BA students of English $<$ $>$ Polish Translation Specialization at the Faculty of English (Adam Mickiewicz University, Poznań). Participants received course credits for taking part in the experiment. They acquired their L2 after the age of five $\left(M_{\text {age of } L 2 \text { acquisition }}=6.24\right.$, $S D=1.44$ ) in the formal school setting in Poland and had not lived in the L2 environment, as a result of which they were classified as late unbalanced Polish-English bilinguals (see de Groot, 2011). All participants were highly proficient in English, as confirmed by the LexTale (Lemhöfer \& Broersma, 2012) results $\left(M_{\text {LexTale score }}=88.63 \%, S D=7.32\right)$.

\section{Materials}

Sentences used in the study were adopted from a database by Jankowiak (2020) and included 60 novel nominal metaphors (e.g., Love is a monastery), 60 novel similes (e.g., Love is like a monastery), and 60 literal sentences (e.g., This monument is a monastery) in Polish and English (see Table 1). The mean sentence lengths of the stimuli ranged from 3 to 5 words in the case of Polish stimuli (nominal metaphors: $M=3.28, S D=.50$; similes: $M=4.28, S D=.48$; literal sentences: $M=4.00$, $S D=.18$ ) and from 3 to 7 in the case of English materials (nominal metaphors: $M=4.12, S D=.82$; similes: $M=5.12, S D=.83$; literal sentences: $M=4.82$, $S D=.66)$. A larger number of words per sentence in English relative to Polish stimuli resulted from the fact that Polish is characterized by a more synthetic structure 
Table 1. Examples of the experimental stimuli (after Jankowiak 2020)

\begin{tabular}{lll}
\hline Novel nominal metaphors & Novel similes & Literal sentences \\
\hline Love is a monastery & Love is like a monastery & This monument is a monastery \\
Memory is a bag & Memory is like a bag & This package is a bag \\
The brain is a wardrobe & The brain is like a wardrobe & This furniture is a wardrobe \\
A descendant is a mirror & A descendant is like a mirror & This surface is a mirror \\
\hline A hangover is a desert & A hangover is like a desert & This area is a desert \\
\hline
\end{tabular}

than English, which is reflected in the higher morpheme-per-word ratio (Jankowiak, 2020).

The database by Jankowiak (2020) provides a list of stimuli which were examined in a series on normative studies that included Likert-type scales on stimuli meaningfulness, familiarity, metaphoricity, and cloze probability. In those normative tests, Polish materials were evaluated by 475 Polish native speakers, while English stimuli by 445 native speakers of English. The meaningfulness of the sentences was evaluated on a scale from 1 (totally meaningless) to 7 (totally meaningful). In Polish, the results of a repeated measures ANOVA showed a main effect of utterance type, $F(3,384)=906.25, p<.001, \epsilon=.774, \eta \mathrm{p}^{2}=.876$. Bonferronicorrected pairwise comparisons showed that literal sentences $(M=5.66, S E=.07)$ were rated as more meaningful than novel similes $(M=4.42, S E=.08), p<.001$, novel similes were rated as more meaningful than novel nominal metaphors $(M=3.87, S E=.08), p<.001$. Similarly, in English, the results of a repeated measures ANOVA showed a main effect of utterance type, $F(3,345)=2026.18, p<.001$, $\epsilon=.872, \eta \mathrm{p}^{2}=.946$, where literal sentences $(M=5.92, S E=.05)$ were rated as more meaningful than novel similes $(M=4.98, S E=.05), p<.001$, novel similes were rated as more meaningful than novel nominal metaphors $(M=4.50, S E=.05)$, $p<.001$ (Jankowiak 2020: 546-551).

The familiarity (i.e., frequency of encountering a stimulus) of the sentences was assessed on a scale from 1 (very rarely) to 7 (very frequently). In Polish, the results of a repeated measures ANOVA showed a main effect of utterance type, $F(2,196)=$ 45.94, $p<.001, \epsilon=.562, \eta \mathrm{p}^{2}=.319$. Bonferroni-corrected pairwise comparisons showed that novel nominal metaphors $(M=1.80, S E=.09)$ were evaluated as less familiar than both novel similes $(M=1.88, S E=.09), p=.014$, and literal sentences $(M=2.51, S E=.13), p<.001$. Furthermore, novel similes were less familiar than literal utterances, $p<.001$. Similarly, in English, the results of a repeated measures ANOVA showed a main effect of utterance type, $F(2,168)=159.86, p<.001$, $\epsilon=.661, \eta p^{2}=.656$, where novel nominal metaphors $(M=2.00, S E=.06)$ were evaluated as less familiar than both novel similes $(M=2.13, S E=.07), p<.001$, and literal sentences $(M=2.92, S E=.10), p<.001$. Furthermore, novel similes were less familiar than literal utterances, $p<.001$ (Jankowiak 2020: 546-551).

Finally, the metaphoricity of the sentences was evaluated on a scale from 1 (very literal) to 7 (very metaphorical). In Polish, the results of a repeated measures ANOVA showed a main effect of utterance type, $F(2,198)=902.18, p<.001$, $\epsilon=.658, \eta \mathrm{p}^{2}=.901$. Bonferroni-corrected pairwise comparisons showed that novel 
similes $(M=5.73, S E=.08)$ were rated as more metaphorical than novel nominal metaphors $(M=5.53, S E=.08), p=.001$, as well as than literal sentences $(M=1.86$, $S E=.07), p<.001$. Additionally, novel nominal metaphors were rated as more metaphorical than literal utterances, $p<.001$. Similarly, in English, the results of a repeated measures ANOVA showed a main effect of utterance type, $F(2,168)=$ 2466.83, $p<.001, \epsilon=.847, \eta \mathrm{p}^{2}=.967$, where novel nominal metaphors $(M=$ $5.85, S E=.04)$ were rated as more metaphorical than novel similes $(M=; 5.61$, $S E=.07), \mathrm{p}=.001$, as well as than literal sentences $(M=1.76, S E=.03)$, $p<.001$. Additionally, novel similes were rated as more metaphorical than literal utterances, $p<.001$ (Jankowiak 2020: 546-551).

For the experiment proper, the stimuli were divided into 3 blocks, each consisting of 20 nominal metaphors, 20 similes, and 20 literal sentences. Each participant was randomly presented with one block only. Translation direction (i.e., L1-L2 vs. L2-L1) was randomized and counterbalanced across participants. Participants were not presented with nominal metaphors and similes sharing the same metaphor source and target domain in order to avoid a potential priming effect (Jankowiak et al., 2021).

\section{Procedures}

The procedures applied in the experiment were in accordance with the ethical guidelines for research with human participants, as recommended and followed by Adam Mickiewicz University, Poznań. Participants were informed about the procedures of the experiment and were asked to sign the informed consent form before the experiment began, in accordance with the Declaration of Helsinki. Also, before the experiment started, each participant was asked to answer questions regarding their demographic information and previous L2 learning experience.

The experiment was conducted in a computer classroom at the Faculty of English, Adam Mickiewicz University, Poznań. Each computer was equipped with Inputlog 8 (Leijten \& Van Waes, 2013), a keylogging software used to record participants' translation process. Inputlog 8 (Leijten \& Van Waes, 2013) was integrated with Microsoft Word and worked unnoticeably in the background, as a result of which the ecological validity of the experiment was not so much compromised.

The experiment was conducted in two consecutive sessions with 13 and 10 participants in each session, respectively, so as to provide a comfortable working environment. Participants were asked to translate a set of 20 nominal metaphors, 20 similes, and 20 literal sentences in the Microsoft Word software, while the Inputlog software was running in the background and recording their translation behavior. No time constraints were imposed in order to eliminate stress connected with time pressure as a factor that could potentially affect participants' decisionmaking in translation. Participants were instructed not to consult the Internet during the experiment, as this might have created a priming effect for the translation equivalents prompted by online dictionaries and other online resources. However, to ensure that participants are familiar with the L2 vocabulary items used in the experiment in the L2-L1 condition, a monolingual glossary was prepared with L2 vocabulary items that were classified in Oxford Advanced Learners' Dictionary 
as $\mathrm{C} 1$ level or higher. Once the experiment finished, participants were asked to complete a LexTale (Lemhöfer \& Broersma, 2012) test.

\section{Results}

The log files of the translation sessions were analyzed by Inputlog 8 (Leijten \& Van Waes, 2013). A general analysis was automatically performed on each log file containing raw data from a translation session per participant, and the XML files were generated. Each file contained every input action performed by a participant on the computer, such as mouse click and movement, letter and function, pauses, total action time, and single time stamps for each action. To obtain the total translation duration of each of the three sets of stimuli (novel nominal metaphors, novel similes, and literal sentences), the time stamps for the beginning of the first sentence and the end of the last sentence translation in a set were computed.

Translation times were analyzed using a mixed ANOVA, with 3 sentence types (novel nominal metaphor vs. novel simile vs. literal sentence) as a within-subject factor and 2 translation directions (forward: L1-L2 vs. backward: L2-L1) as a between-subject factor. Additionally, LexTale scores and participants' AoA values were included as covariates in the analyses. Significance values for pairwise comparisons were corrected for multiple comparisons using the Bonferroni correction. When Mauchly's tests showed that the assumption of sphericity was violated, the Greenhouse-Geisser correction was applied, and the original degrees of freedom were reported with the corrected $p$ value.

The results of the mixed ANOVA showed an interaction between sentence type and translation direction, $F(2,38)=4.53, p=.017, \eta_{\mathrm{p}}^{2}=.193$. First, a post hoc univariate general linear model was conducted with each sentence type as a dependent variable and translation direction as a fixed factor, which showed a statistically significant main effect of translation direction for literal sentences, $F(1,19)=4.67$, $p=.044, \eta_{\mathrm{p}}^{2}=.197$, whereby they evoked longer translation duration in the L1-L2 relative to the L2-L1 translation direction. Further analyses were performed individually for each translation direction employing repeated measures ANOVAs. The analysis for L2-L1 translation direction revealed a main effect of sentence type, $F(2,16)=9.66, p=.002, \eta_{\mathrm{p}}{ }^{2}=.547$. Pairwise comparisons further showed that novel nominal metaphors elicited significantly longer translation times than both novel similes, $p=.042$, and literal sentences, $p=.009$. There was no statistically significant difference between novel similes and literal utterances, $p>.05$. The analysis for the L1-L2 translation direction, on the other hand, revealed no statistically significant effect of sentence type, $p>.05$. Mean translation times per sentence of each sentence type in the two translation directions are provided in Figure 1.

In addition to the interaction between sentence type and translation direction, the main mixed ANOVA also yielded a main effect of sentence type, $F(2,38)=$ $6.43, p=.004, \eta_{\mathrm{p}}^{2}=.253$. Pairwise comparisons further showed that novel nominal metaphors required significantly longer translation times than both novel similes, $p=.008$, and literal sentences, $p=.026$. There was no statistically significant difference between novel similes and literal utterances, $p>.05$. Furthermore, the analysis showed a graded effect across the sentence type, with the shortest translation times 


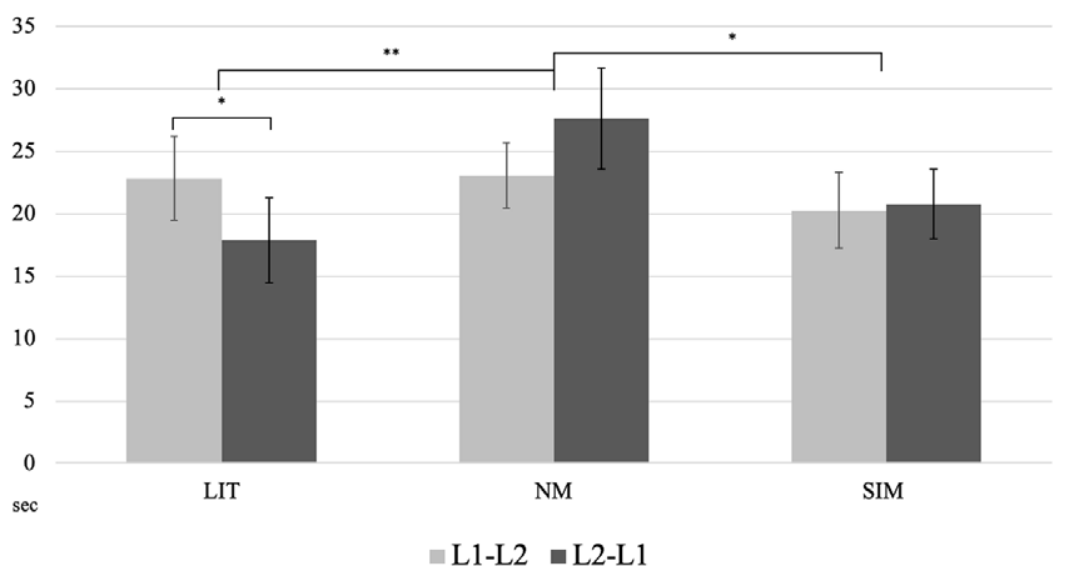

Figure 1. Mean translation times (in seconds) for literal sentences (LIT), novel nominal metaphors (NM), and novel similes (SIM) in each translation direction (L1-L2, L2-L1). Whiskers represent 95\% confidence intervals and significant differences between different conditions are marked with asterisks.

for literal sentences, followed by novel similes, and finally novel nominal metaphors, $F(1,19)=8.58, p=.009, \eta_{\mathrm{p}}{ }^{2}=.311$. There was no statistically significant effect of translation direction, $p>.05$. Also, there was no effect of either the LexTale scores or AoA values on the dependent measures, $p s>.05$.

\section{Discussion}

The present study was aimed to examine the keystroke correlates of novel meaning translation and to investigate the role of translation direction (i.e., L1-L2 vs. L2-L1) in this process. Additionally, the study exploratively examined the role of a linguistic structure of a novel meaning (i.e., novel nominal metaphors vs. novel similes) in the process of translation. To this end, Polish (L1) - English (L2) translation students were asked to translate novel nominal metaphors $(A$ is $B)$, novel similes ( $A$ is like $B)$, and literal sentences in either the L1-L2 or L2-L1 translation direction, while their translation time was measured using the keystroke logging method.

First of all, the results showed a general effect of sentence type, whereby novel nominal metaphors elicited longer translation times than both novel similes and literal sentences. The present findings might be, therefore, suggestive of an increased cognitive effort engaged in translating novel nominal metaphors relative to novel similes, thus showing that novel similes were easier and faster to translate. Importantly, such results might be interpreted within the assumptions of the Career of Metaphor Model (Bowdle \& Gentner, 2005), according to which novel meaning processing requires comparison mechanisms, and should consequently be facilitated when novel meanings are already presented in a comparison form (i.e., a form of a simile), which automatically initiates comparison mechanisms. Nominal metaphors, on the other hand, need to be converted into a comparison 
form so as to activate comparison mechanisms (Bowdle \& Gentner, 2005), which might be reflected in faster translation of similes compared to nominal metaphors. Though the crucial role of comparison mechanisms in novel metaphor processing has been previously confirmed in studies on meaning comprehension (e.g., Jankowiak et al., 2021; Lai \& Curran, 2013; Shibata et al., 2012; but see Glucksberg \& Haught, 2006), the present study provides a tentative support to this assumption also in the context of meaning translation, which has thus far been under-investigated.

At the same time, a similar time course of novel simile and literal sentence translation might come as a surprise, since previous studies have pointed to fact that the mapping between the dissimilar concepts necessary for novel meaning (including novel simile) processing is more resource-intensive than mapping between the similar concepts, which is the case in literal meaning processing (Jankowiak et al., 2021; Lai \& Curran, 2013; Shibata et al., 2012). Also, previous comprehension studies have repeatedly showed that the comprehension of meanings that are novel, and thus high in metaphoricity, is generally more cognitively taxing than literal utterances (e.g., Arzouan et al., 2007; Jankowiak et al., 2017; Lai et al., 2009; Obert et al., 2018). The similar amount of time invested in translating novel similes and literal utterances might nonetheless indicate that the comparison mechanisms evoked when processing novel similes might have facilitated their translation to the point where they were perceived as more conventional meanings, and thus their translation was no longer so effortful. More research is, nevertheless, needed in order to provide more insights into the role of metaphor conventionality in the translation process.

The present study might thus be interpreted within the frameworks based on the comparison view of metaphor processing (but see categorization-based views on metaphor processing; Glucksberg \& Keysar, 1990; Glucksberg et al., 1997; Glucksberg \& Haught, 2006). Comparison-based theories (Gentner, 1983; Gentner \& Markman, 1997; Gentner \& Wolff, 1997; Gentner \& Bowdle, 2001; Gentner et al., 2001) predict that novel metaphor comprehension involves the literal meaning of metaphor source to be structurally aligned with metaphor target, and they specify that not all that is true of the source concept is true of the target concept. Conventional (familiar) metaphor processing, on the other hand, is assumed to be based on the categorization process that involves a linear alignment between metaphor source and target, where all that is true of the source domain is true in the target domain (Lai \& Curran, 2013). Consequently, much as categorization processes are more rapid and automatic in the case of conventional metaphors (Jankowiak, 2019), they are postulated to be more resource-intensive in the case of novel (i.e., highly creative and metaphorical) meaning processing. Namely, for novel metaphor processing, the linear alignment involved in the categorization process is inapplicable, and therefore, comparison mechanisms have to be initiated (Bowdle \& Gentner, 2005; Goldstein et al., 2012). In line with these predictions, the simulation experiment based on the Semantic Space Model (Utsumi, 2011), which is based on word co-occurrence and allows for a computation of semantic similarity between lexical items, confirmed that semantically diverse meanings (i.e., novel metaphors) are easier to be comprehended when the novel meaning is presented in a simile form, thus further supporting the comparison view of novel metaphor processing. 
Importantly, the present findings show that the effects previously observed in meaning comprehension studies might be replicated in translation studies, which is interesting, as while comprehension studies mostly tap into automatic mechanisms engaged in meaning activation and integration, translation tasks additionally require conscious information processing, decision-making and problem-solving, attention control, and self-monitoring. It thus seems that cognitive mechanisms engaged in translating novel utterances might be modulated by the linguistic structure, which can facilitate the activation of comparison mechanisms, thus assisting the translation process. Furthermore, longer translation times for novel nominal metaphors than literal utterances are potentially in line with the Cognitive Translation Hypothesis (Mandelblit, 1995), as well as previous studies employing the keystroke logging technique, which have also shown an increased difficulty in metaphor translation, as reflected in longer translation duration (e.g., Jakobsen et al., 2007; Lehka-Paul, 2020). A different time course of novel nominal metaphor and literal sentence translation might potentially provide support to previous comprehension studies showing that nonliteral language comprehension requires establishing the correspondence between distinct knowledge domains, as opposed to literal meanings that involve concepts similar to one another (Bowdle \& Gentner, 2005; Cacciari \& Glucksberg, 1994). At the same time, however, the stimuli used in the present study were not analyzed and matched on whether they share the same or different domains when translated into Polish and English, and therefore, further research into this notion seems necessary in order to show whether the translation process is modulated by domain specificity.

Interestingly, in addition to the main effect of utterance type, we found an interaction between sentence type and translation direction, whereby the effect for novel similes relative to novel nominal metaphors was observed only in the backward (L2-L1) translation direction, and it disappeared in the forward (L1-L2) direction. The effect observed in the $\mathrm{L} 2-\mathrm{L} 1$ translation direction might be interpreted in terms of a facilitated re-mapping process (see Massey, 2016) when translating metaphoric meanings into the native language. Thus, it seems that while cross-domain mappings necessary for metaphor comprehension are generally language-independent, the translation of this mapping into a target language might be more difficult when the access to semantic representations is hindered (i.e., in the forward translation). Also, the obtained findings lend support to the idea that production advantage in L1 hinders the L1-L2 translation process (Kelly, 2005). Furthermore, the lack of effect of utterance type in the forward translation direction seems to be in line with the postulates of the Revised Hierarchical Model (Kroll \& Stewart, 1994), according to which access to semantic representations is less automatic when translating into the non-native language, whose lexical-level representations are assumed to have weaker connections with conceptual-level representations. Interestingly, we also observed a trend whereby novel metaphors were translated faster into participants' L2 than into their L1, which might have resulted from general differences in participants' sensitivity to L1 vs. L2. Namely, it might have been easier for participants to produce a translation of an unfamiliar meaning in L2 than in L1, since translation into their native tongue may have required more strategic and conscious choices regarding the best possible translation of a novel meaning. As sensitivity to the conceptual representations might be generally lower in L2, this might be 
reflected in faster, yet potentially less accurate translations. This issue would, however, need to be investigated in further studies into novel metaphor translation that additionally accounts for translation performance and its accuracy.

Interestingly, despite the aforementioned interaction, the present results yielded no main effect of translation direction. A generally similar translation duration in both translation directions might result from a proficiency level of participants tested, who were all highly proficient in English as their second language, as confirmed by the LexTale results (Lemhöfer \& Broersma, 2012). Such findings are in line with the tenets postulated within the concept mediation assumption of the Revised Hierarchical Model (Kroll \& Stewart, 1994), which argues for a direct access to meaning representations from both L1 and L2 lexical levels of representation when bilingual speakers are proficient in their L2, as a result of which both L1-L2 and L2-L1 translation directions are similarly automatic. The findings are also in accordance with recent translation studies (da Silva et al., 2017; Whyatt, 2019) that suggest that translation duration may not be influenced by directionality when translators are highly skilled in the translation task.

It, however, needs to be noted that the obtained results do not necessarily provide a full picture of the research question under investigation, as the data analyses were based only on one behavioral indicator, that is, translation duration. Moreover, the keystroke logging technique is a method that might not be sensitive enough to reveal exact differences in cognitive mechanisms engaged in creative language translation. Namely, keylogging data are only able to reveal information about the translator's typing process, while other processes involved in translation, such as reading and information search, might also elicit important details about the ways metaphoric utterances are processed and translated into both directions. Hence, future studies would benefit from data triangulation, that is, the use of different data collection methods to explore a research question. For example, eye-tracking data would allow researchers to identify the areas of the text (e.g., point to the specific sentences or parts of sentences) that were particularly taxing in terms of cognitive effort involved in their processing and/or describe the translator's reading behavior (see Sjørup, 2008; 2013). Combining eye-tracking and keylogging data might thus give a more comprehensive picture of the processes involved in the translation of metaphoric and literal utterances in both translation directions. Finally, future studies should also analyze the quality of translations produced, so as to see how different translation behaviors correlate with the final product of translation.

Furthermore, the present results might have been influenced by the stimuli used in the translation task. Namely, while the stimuli have been well matched on a number of lexical and semantic variables (Jankowiak 2020), the two figurative conditions (i.e., novel similes and novel nominal metaphors) - due to their high degree of creativity - were not matched with literal sentences on the meaningfulness, metaphoricity, and familiarity levels. Consequently, the stimuli used in the literal condition might have been faster to translate compared to novel nominal metaphors due to being more meaningful, literal, and/or familiar. Even though matching novel figurative utterances on those variables might be challenging, future studies should account for how these factors contribute to translation difficulty (see Blasko \& Connine, 1993; Cardillo et al., 2012; Diaz et al., 2011; Forgács et al., 2014). 
Finally, the literal sentences used in the present study were phrased as demonstrative structures that use a demonstrative determiner this, and these structures are assumed to require a more extended context to facilitate meaning processing (see Kroeger, 2018). Since the stimuli used in the study were all presented as isolated statements, participants might have perceived literal utterances as less natural and meaningful. Though the stimuli used in the study were previously pretested in a series of normative tests (Jankowiak, 2020), which showed that in both Polish and English literal utterances were evaluated as significantly more meaningful than both nominal metaphors and similes, future research should further examine the process of demonstrative sentence translation by, for instance, including a condition of literal comparisons (e.g., A purse is like a bag).

\section{Conclusion}

To the best of our knowledge, the present study was the first attempt to investigate novel meaning translation, as modulated by translation direction. First of all, the obtained results showed longer translation durations for novel nominal metaphors relative to literal utterances, thus confirming that novel metaphor translation is more cognitively taxing than literal meaning translation. Furthermore, we found faster translation times for novel similes than novel nominal metaphors, yet only in the L2-L1 translation direction. These results indicate that comparison mechanisms, which, unlike in the case of similes, are not automatically initiated when processing nominal metaphors increase the difficulty of novel meaning translation. However, such an effect is stronger in the L2-L1 translation probably due to a facilitated access to semantic representations in this translation direction. In addition, the absence of the main effect of directionality on translation duration is in line with recent directionality research in translation studies (da Silva et al., 2017; Whyatt, 2019) and suggests that the process of translation may be comparatively demanding in both directions when participants are highly proficient bilinguals.

Funding acknowledgement. Katarzyna Jankowiak is supported by the Foundation for Polish Science

\section{References}

Arzouan, Y., Goldstein, A., \& Faust, M. (2007). Brainwaves are stethoscopes: ERP correlates of novel metaphor comprehension. Brain Research, 1160, 69-81.

Bayer-Hohenwarter, G. (2009). Comparing translational creativity scores of students and professionals: flexible problem-solving and/or fluent routine behaviour? In S. Göpferich, A. L. Jakobsen, \& I. M. Mees (Eds.), Behind the mind: Methods, Models and Results in Translation Process Research (pp. 83-112). Copenhagen: Samfundslitteratur.

Blasko, D. G., \& Connine, C. M. (1993). Effects of familiarity and aptness on metaphor processing. Journal of Experimental Psychology: Learning, Memory and Cognition, 19(2), 295-308.

Bowdle, B. F., \& Gentner, D. (2005). The Career of Metaphor. Psychological Review, 112(1), $193-216$.

Buchweitz, A., \& Alves, F. (2006). Cognitive adaptation in translation: An interface between language direction, time and recursiveness in target text production. Letras de Hoje, 41(2), 241-272.

Cacciari, C., \& Glucksberg, S. (1994). Understanding figurative language. In M. A. Gernsbacher (Ed.), Handbook of psycholinguistics (pp 447-477). Academic Press.

Cardillo, E. R., Watson, C. E., Schmidt, G. L., Kranjec, A., \& Chatterjee, A. (2012). From novel to familiar: tuning the brain for metaphors. Neuroimage 59, 3212-3221. 
Carl, M, (2012). Translog - II: A program for recording user activity data for empirical reading and writing research. In N. Calzolari, K. Choukri, T. Declerck, M. U. Dogan, B. Maegaard, J. Mariani, A. Moreno, J. Odijk \& S. Piperidis (Eds.), Proceedings of the Eight International Conference on Language Resources and Evaluation (pp. 4108-4112). Istanbul: European Language Resources Association (ELRA).

Conklin, K., \& Pellicer-Sánchez, A. (2016). Using eye-tracking in applied linguistics and second language research. Second Language Research, 1-15.

da Silva, I. A., Alves, F., Schmaltz, M., Pagano, A., Wong, D., Chao, L., Leal, A. L., Quaresma, P., Garcia, C., \& da Silva, G. E. (2017). Translation, post-editing and directionality: A study of effort in the ChinesePortuguese language pair. In A. L. Jakobsen, \& B. Mesa (Eds.). Translation in Transition (pp. 108-134). Amsterdam: John Benjamins.

de Groot, A. M. B. (2011). Bilingual Cognition: An Introduction. Psychology Press.

Diaz, M. T., Barrett, K. T., \& Hogstrom, L. J. (2011). The influence of sentence novelty and figurativeness on brain activity. Neuropsychologia 49, 320-330.

Ferreira, A. (2012). Investigando o processamento cognitivo de tradutores profissionais em tradução direta e inversa no par linguístico inglês português. Cadernos de Tradução, 29, 73-92.

Fonseca, N. B. (2015). Directionality in translation: Investigating prototypical patterns in editing procedures. Translation \& Interpreting, 7(1), 111-125.

Forgács, B., Lukács, A., \& Pléh, C. (2014). Lateralized processing of novel metaphors: disentangling figurativeness and novelty. Neuropsychologia, 56, 101-109.

Gentner, D. (1983). Structure-mapping: A theoretical framework for analogy. Cognitive Science 7(2), $155-170$.

Gentner, D., \& Bowdle, B.F. (2001). Convention, form, and figurative language processing. Metaphor and Symbol, 16(3), 223-247.

Gentner, D., Bowdle, B.F., Wolff, P., \& Boronat, C. (2001). “Metaphor is like analogy”. In Dedre Gentner, Keith J. Holyoak \& Boicho N. Kokinov (Eds.), The analogical mind: Perspectives from cognitive science (pp. 199-253). Cambridge: MIT Press.

Gentner, D., \& Markman, A.B. (1997). Structure mapping in analogy and similarity. American Psychologist, 52(1), 45-56.

Gentner, D., \& Wolff, P. (1997). Alignment in the processing of metaphor. Journal of Memory and Language, 37(3), 331-355.

Gibbs, J., \& Colston, H. L. (2012). Interpreting Figurative Meaning. Cambridge University Press.

Glucksberg, S., \& Haught, C. (2006). On the relation between metaphor and simile: When comparison fails. Mind and Language, 21(3), 360-378.

Glucksberg, S., \& Keysar, B. (1990). Understanding metaphorical comparisons: Beyond similarity. Psychological Review, 97(1), 3-18.

Glucksberg, S., Manfredi, D.A., \& McGlone, M.S. (1997). Metaphor comprehension: How metaphors create new categories. In T. B. Ward, S. M. Smith \& J. Vaid (Eds.), Creative thought: An investigation of conceptual structures and processes (pp. 327-350). Washington, DC: American Psychological Association.

Goldstein, A., Arzouan, Y., \& Faust, M. (2012). Killing a novel metaphor and reviving a dead one: ERP correlates of metaphor conventionalization. Brain and Language, 123(2), 137-142.

Göpferich, S. (2008). Translationsprozessforschung. Stand, Methoden, Perspektiven. Tübingen: Narr.

Hagoort, P., Hald, L., Bastiaansen, M., \& Petersson, K.M. (2004) Integration of word meaning and world knowledge in language comprehension. Science, 16, 438-441.

Heredia, R. R., \& Cieślicka, A. B. (2016). Metaphoric reference: An eye movement analysis of SpanishEnglish and English-Spanish bilingual readers. Frontiers in Psychology, 7.

Jakobsen, A. L., Jensen K. T. H., \& Mees I. M. (2007). Comparing modalities: Idioms as a case in point. In F. Pöchhacker, A. L. Jakobsen, \& I. M. Mees (Eds.), Interpreting Studies and Beyond. A Tribute to Miriam Shlesinger (pp. 217-249). Copenhagen: Samfundslitteratur.

Jankowiak, K. (2019). Lexico-semantic processing in bilingual figurative language comprehension: Behavioral and electrophysiological evidence. Poznań: Wydawnictwo Naukowe UAM.

Jankowiak, K. (2020). Normative Data for Novel Nominal Metaphors, Novel Similes, Literal, and Anomalous Utterances in Polish and English. Journal of Psycholinguistic Research, 49, 541-569.

Jankowiak, K., Naranowicz, M., \& Rataj, K. (2021). Metaphors are like lenses: Electrophysiological correlates of novel meaning processing in bilingualism. International Journal of Bilingualism, 25(3), $668-686$. 
Jankowiak, K., Rataj, K., \& Naskręcki, R. (2017). To electrify bilingualism: Electrophysiological insights into bilingual metaphor comprehension. PloS One, 12(4), 1-30.

Kelly, D. (2005). A handbook for translator trainers. Manchester: St. Jerome Publishers.

Kroeger, P. (2018). Analyzing meaning: An introduction to semantics and pragmatics. Dallas: Language Science Press.

Kroll, J. F., \& Stewart, E. (1994). "Category interference in translation and picture naming: Evidence for asymmetric connections between bilingual memory representations. Journal of Memory and Language, 33, $149-174$.

Lai, V. T., \& Curran, T. (2013). ERP evidence for conceptual mappings and comparison processes during the comprehension of conventional and novel metaphors. Brain and Language, 127(3), 484-496.

Lai, V. T., Curran, T., \& Menn, L. (2009). Comprehending conventional and novel metaphors: An ERP study. Brain Research, 1284, 145-155.

Lehka-Paul, O. (2020). Personality matters. The translator's personality in the process of self-revision. Leuven: Leuven University Press.

Leijten, M., \& Van Waes, L. (2013). Keystroke logging in writing research: Using Inputlog to analyze and visualize writing processes. Written Communication, 30(3), 358-392.

Lemhöfer, K., \& Broersma, M. (2012). Introducing LexTALE: A quick and valid Lexical Test for Advanced Learners of English. Behavior Research Methods, 44(2), 325-343.

Mandelblit, N. (1995). The cognitive view of metaphor and its implications for translation theory. Translation and Meaning, 3, 483-495.

Massey, G. (2016). Remapping Meaning: Exploring the Products and Processes of Translating Conceptual Metaphor. In Ł. Bogucki, B. Lewandowska-Tomaszczyk \& M. Thelen (Eds.), Translation and Meaning. New Series, Vol. 2 (pp. 67-83). Frankfurt am Main: Peter Lang.

Massey, G., \& Ehrensberger-Dow, M. (2017). Translating conceptual metaphor: The processes of managing interlingual asymmetry. Research in Language, 15(2), 173-189.

Newmark, P. (1988). A Textbook of Translation. Hertfordshire: Prentice Hall.

Ni, W., Constable, R. T., Mencl, W. E., Pugh, K. R., Fulbright, R. K., Shaywitz, S. E., Shaywitz, B. A., Gore, J. C. \& Shankweiler, D. (2000) An event-related neuroimaging study distinguishing form and content in sentence processing. Journal of Cognitive Neuroscience, 12, 120-133.

Obert, A., Gierski, F., \& Caillies, S. (2018). He catapulted his words from the dais: An ERP investigation of novel verbal metaphors. Journal of Neurolinguistics, 47, 59-70.

Pavlović, N. (2007). Directionality in collaborative translation processes. A study of novice translators. $(\mathrm{PhD}$ Dissertation). Tarragona: Universitat Rovira i Virgili.

Pavlović, N., \& Jensen, K. T. H. (2009). Eyetracking translation directionality. In A. Pym \& A. Perekrestenko (Eds.), Translation Research Projects 2 (pp. 93-109). Tarragona: Intercultural Studies Group.

Potter, M. C., So, K.-F., von Eckardt, B., \& Feldman, L. B. (1984). Lexical and conceptual representation in beginning and proficient bilinguals. Journal of Verbal Learning and Verbal Behavior, 23(1), 23-38.

Reiss, K. (2000). Type, kind and individuality of text: Decision making in translation. (S. Kitron, Trans.). In L. Venuti (Ed.), The Translation Studies Reader (pp. 160-171). London: Routledge.

Schäffner, C. (2004). Metaphor and translation: Some implications of a cognitive approach. Journal of Pragmatics, 36, 1253-1269.

Shibata, M., Toyomura, A., Motoyama, H., Itoh, H., Kawabata, Y., \& Abe, J. (2012). Does simile comprehension differ from metaphor comprehension? A functional MRI study. Brain and Language, 121(3), 254-260.

Sjørup, A. C. (2008). Metaphor comprehension in translation: Methodological issues in a pilot study. In S. Göpferich, A. L. Jakobsen, \& I. M. Mees (Eds.), Looking at Eyes. Eye-Tracking Studies of Reading and Translation Processing (pp. 53-77). Copenhagen: Samfundslitteratur.

Sjørup, A. C. (2013). Cognitive Effort in Metaphor Translation. An Eye-tracking and Keylogging Study. Doctoral dissertation. Copenhagen: Copenhagen Business School.

Stevens, J. P. (2009). Applied multivariate statistics for the social sciences. Mahwah, NJ: Lawrence Erlbaum Associates, Publishers.

Toury, G. (1995). Descriptive Translation Studies - and Beyond. Amsterdam and Philadelphia: John Benjamins.

Utsumi, A. (2011). Computational exploration of metaphor comprehension processes using a semantic space model. Cognitive Science, 35(2), 251-296. 
Vaid, J., López, B. G., \& Martínez, F. E. (2015). Linking the Figurative to the Creative: Bilinguals' Comprehension of Metaphors, Jokes, and Remote Associates. In R. R. Heredia \& A. B. Cieślicka (Eds.), Bilingual Figurative Language Processing (pp. 53-86). Cambridge University Press.

Wang, X., \& Jankowiak, K. (2021). "Electrophysiological insights into the role of proficiency in bilingual novel and conventional metaphor processing", Linguistic Approaches to Bilingualism.

Whyatt, B. (2019). In search of directionality effects in the translation process and in the end product. Translation, Cognition \& Behavior, 2(1), 79-100.

Cite this article: Jankowiak, K. and Lehka-Paul, O. (2022). Novel metaphor translation is modulated by translation direction. Applied Psycholinguistics 43, 177-192. https://doi.org/10.1017/S0142716421000461 